\title{
Hole Reshaping Routing in Large-Scale Mobile Ad-Hoc Networks
}

\author{
Peiqiang $\mathrm{Li}^{\dagger}$, Guojun Wang ${ }^{\dagger \ddagger}$ and Jie $\mathrm{Wu}{ }^{\ddagger}$ \\ ${ }^{\dagger}$ School of Information Science and Engineering \\ Central South University \\ $\ddagger$ Department of Computer Science and Engineering \\ Changsha 410083, China \\ Florida Atlantic University \\ Boca Raton, FL 33431, USA
}

\begin{abstract}
Mobile ad-hoc networks (MANETs) usually contain sparse or even empty regions called holes. The local optimum problem will occur when routing packets meet holes in the network. In this paper, we propose a novel hole-reshaping routing protocol (HRR) in large-scale MANETs. It effectively solves the hole problem by regularizing a hole with an ellipse, and then locally broadcasting the hole information away from the hole. Simulation results show that the proposed protocol guarantees finding a short routing path with a small routing delay, which is a prerequisite to achieve scalability in large-scale networks.
\end{abstract}

Index Terms-mobile ad-hoc networks, local optimum, hole shadowing routing, hole reshaping routing

\section{INTRODUCTION}

A mobile ad-hoc network is a particular type of wireless network in which an association of mobile nodes forms a multi-hop radio network in a decentralized manner without any support of a fixed infrastructure. Each mobile node acts as both a terminal and a router, and the control of the network activities is distributed to all nodes in the network. Mobile nodes communicate over wireless links that typically have less bandwidth than wired networks. In addition, the network topology is generally dynamic because the connectivity among nodes may change with time due to node mobility, radio communication nature, and power limitations. These features of MANETs have posed a lot of challenges in designing an effective, reliable, and scalable routing protocol.

At present, the geographic routing protocols have attracted more and more attention and are proposed to solve the scalability problem in MANETs. In geographic routing, it is assumed that each node knows its own location and the location of destination nodes. Using this location information, the source and each intermediate node select the next hop closest to the destination. This routing technique is known as greedy next hop selection. Geographic routing may operate like a reactive routing scheme. It searches for a route to the destination when a packet to the destination is generated. However, unlike the general reactive routing schemes such as AODV[1] and DSR[2], geographic routing does not flood route request packets. Because each intermediate node makes routing decisions independently based on location information. Geographic routing is scalable and can be applied to largescale mobile ad-hoc networks.
The geographic greedy forwarding [3], [4], as a simple, efficient, and scalable strategy, is the most promising routing scheme in geographic routing. But due to sparse deployment, physical obstacles, node failures, communication jamming, power exhaustion, and animus interference [5]-[8], holes may exist. When routing packets meet holes and the forwarding process is blocked at a node, called a dead-end node, whose neighbors are all farther away from the destination than itself in the network, the greedy forwarding usually meets the local optimum problem. So, in addition to greedy forwarding, some schemes [3], [4], [9], [10] have been proposed to solve this problem. But most of the proposed solutions are originated from the face routing on planar graphs. Routing that is performed in order to escape from dead-end nodes is called a fallback routing. A packet enters the fallback routing mode when it arrives at a dead-end node and the packet resumes greedy forwarding when it reaches a node that is closer to the destination than the dead-end nodes.

Even though previously proposed geographic routing algorithms get around communication holes and satisfy the acceptable packet delivery ratios, they may select a long detouring paths because they do not know the shape of the hole. When a node meets the local optimum problem, most fallback routing protocols blindly select a left or right detour path according to pre-defined rules such as the right hand rule. As a result, they may select a long detour path even when there are much shorter paths in the other direction. This problem is known as the blind detouring problem [15]. On the side, these fallback routing protocols get away from the hole, when the data packet reaches the dead-end node. But if we know the information of the hole in advance, we can select shorter paths by pre-bypassing the hole. This problem is named the triangular routing problem [15]. We will give a detailed description in section II.

Regarding all the above mentioned problems, we propose a novel hole-reshaping routing protocol (HRR) in large-scale MANETs. The main idea is as follows: It firstly regularizes the hole with an ellipse, and then sends the information of the hole to all nodes in the hole boundary. When these boundary nodes receive the information, they again locally broadcast the information to $h$ hops outside. Here, we call the range of $h$ hops the shadow of the hole. Finally, all nodes in the shadow 


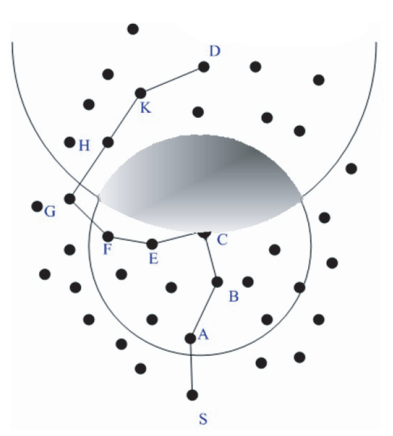

Fig. 1. The local optimum problem

of the hole know the information of the hole. Due to knowing the information of the hole, when a data packet reaches the range of the shadow of the hole, it will decide which side of the hole is better to forward the packet. So, this effectively solves the triangular routing problem and the blind detouring problem.

To sum up, the main motivation of our work is to shorten the path length and reduce the routing delay when some holes exist between the source node and the destination node. In the end of this paper, we evaluate the performance of HRR. We observed that HRR always performs better than the other geographic routing protocols. Furthermore, HRR provides a shorter path and a lower routing delay than other protocols. The simulation results also show that HRR runs well in largescale mobile networks.

The rest of this paper is structured as follows. In Section II, we review some geographic routing protocols and describe some related works. Section III provides the detailed description of the proposed protocol. In Section IV, we evaluate the performance of the proposed protocol by simulation analysis. Concluding remarks are given in Section V.

\section{RELATED WORKS}

With the in-depth study of routing protocols in MANETs, the geographic routing protocols become a hot research topic. Currently, there are many geographic routing protocols such as GPSR [9], HSR [10], LARGER [11] and SLGF [12]. All of them are based on the non-state greedy routing, without the need to maintain any routing table. In GPSR, each node knows its own location and the location of the destination node. Using this location information, the source and each intermediate node can easily select the next hop node closest to the destination node. GPSR usually adopts two routing methods: greedy routing and perimeter routing. At first, GPSR transmits the data packet by greedy routing, but when it meets the local optimum problem, it has to send the data packet by perimeter routing.

As shown in Fig. 1, $D$ is the center of the semicircle, and the distance between $D$ and $C$ is the radius of the semicircle. The circle whose center is $C$ represents the 1 hop transmission range of $C$. Here, $S$ is the source node and $D$ is the destination node. At first, $S$ sends the data packet by greedy routing,

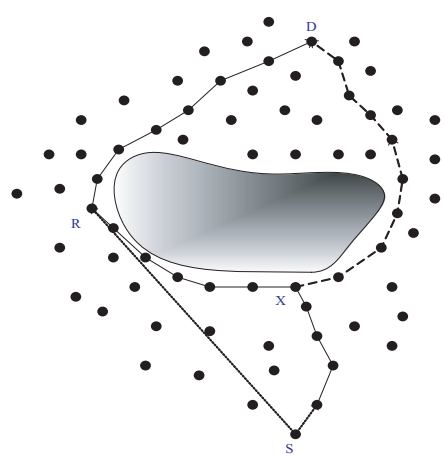

Fig. 2. The triangular routing problem and the blind detouring problem

but when the data packet reaches the intermediate node $C$, we may see from Fig. 1 that there is a path $S A B C E F G H K D$ between $S$ and $D$. However, in all neighbor nodes of $C$, the distance between $C$ and $D$ is the shortest. So $C$ cannot make progress by greedy routing, that is, the data packet can not reach the destination node $D$. Regarding this problem, Karp and Kung [9] propose a perimeter routing algorithm that looks for the next hop along the border of the void area. In Fig. 1, e.g., it will choose $E$, and then $E$ determines whether it can reach $D$ by greedy routing, if it can not reach $D$, it will again look for the next hop by perimeter routing until a node can reach $D$ by greedy routing. However, we should know that it must construct planar graphs to describe the network topology before implementing the perimeter routing. Nowadays, there are some algorithms to do so, such as relative neighborhood graph (RNG) [13] and Gabriel graph (GG) [14].

However, there are still some problems, as shown in Fig. 2 , after $S$ forwards a data packet to $X$ in a greedy way, it will meet the local optimum problem, and can not forward the data to $D$. At this point, GPSR will send the data packet to $R$ by perimeter routing until it reaches $D$. The solid line is the path from $S$ to $D$ selected by GPSR that applies the right hand rule, but due to the path $S$ - $R$ being shorter than the path $S-X-R$, we may select the shortest path $S-R-D$. This is the triangular routing problem. In addition, when the data reaches $X$, if we select the next hop from the right side of $X$, it will find a shorter path than the detour path $S-X-R-D$. So there is another problem, called the blind detouring problem.

In HSR, the local information of the hole is broadcasted to $h$ hops outside. When the node forwarding the packet is in the range of the $h$ hops, it can select a node from its neighbor nodes that is not in the direction of the hole and the nearest node from the destination node. It is seen to some extent that HSR solves the triangular routing problem, but because HSR does not know the overall information of a hole, it still does not deal with the blind detouring problem well.

In SLGF, an information model for geographic greedy forwarding in mobile ad-hoc sensor networks is proposed. Under this information model, the hole and its affected area are identified easily and quickly in an unsafe area and the unsafe 
area can also be estimated as a rectangular region in the local view of unsafe nodes. With such estimate information, when a data packet reaches an unsafe node $u, u$ can rotate the ray $u d$ in counter-clockwise order to find the first node $v$ safe to $d$, and then send the packet to such a node $v$ by perimeter routing. In this way, SLGF can solve the local optimum problem well. However, because SLGF knows only the estimated shape about an unsafe area, it can not solve the blind detouring problem well.

All the geographic routing protocols mentioned above will meet the triangular routing problem and the blind detouring problem. Regarding some problems, we propose a novel holereshaping routing protocol. It effectively solves these problems by reshaping the hole with an ellipse and locally broadcasting the hole information away from the hole. In section III, we will give more description to the proposed routing protocol.

\section{THE PROPOSED PROTOCOL}

\section{A. Network Model}

In this paper, we assume the network is divided into many square regions and the side length for each square region is $d$. Each square region is an independent grid. There are two heterogeneous nodes: ordinary nodes and super nodes. Each node is uniform and knows its own location by GPS. Each ordinary node has a short radio whose communication radius $R$ is $\sqrt{2} d$, and in charge of communication between the adjacent cluster members. The super node has the short radio and the long radio whose communication radius $R$ is $2 \sqrt{2} d$ and is responsible for the communication between the neighbor cluster heads (CHs). The $\mathrm{CHs}$ are selected from super nodes. When a $\mathrm{CH}$ is selected in a grid, the remaining super nodes and ordinary nodes all become cluster members.

The whole network is divided into two layers: the mobile node layer and the backbone layer. The mobile node layer consists of all mobile nodes, and the backbone layer consists of $\mathrm{CHs}$ in each grid. When a $\mathrm{CH}$ alternately changes, and there are still some super nodes in each cluster, the backbone layer does not change.

In this heterogeneous network, each $\mathrm{CH}$ periodically maintains a cluster routing table, which saves the information of its cluster members and its adjacent $\mathrm{CHs}$. The maintenance process about the cluster routing table is as follows:

Each cluster member periodically broadcasts a message with its global ID number, geographic location, and current time stamp to its $\mathrm{CH}$, then when the $\mathrm{CH}$ receives the message, it computes the cluster routing table, which contains all routing messages to every cluster member, and then broadcasts this message to each cluster member. In addition, the routing table also contains the information of direct neighbor $\mathrm{CHs}$ obtained by exchanging the message with its neighbor $\mathrm{CHs}$. If a $\mathrm{CH}$ has not received any of its members or neighbor $\mathrm{CH}$ information for a certain time, it will think the cluster member or head has left, and thus delete the information from the routing table. When a $\mathrm{CH}$ leaves its grid, it will send the routing table information to the candidate $\mathrm{CH}$. In this paper, we assume the super nodes in the network are uniformly and randomly

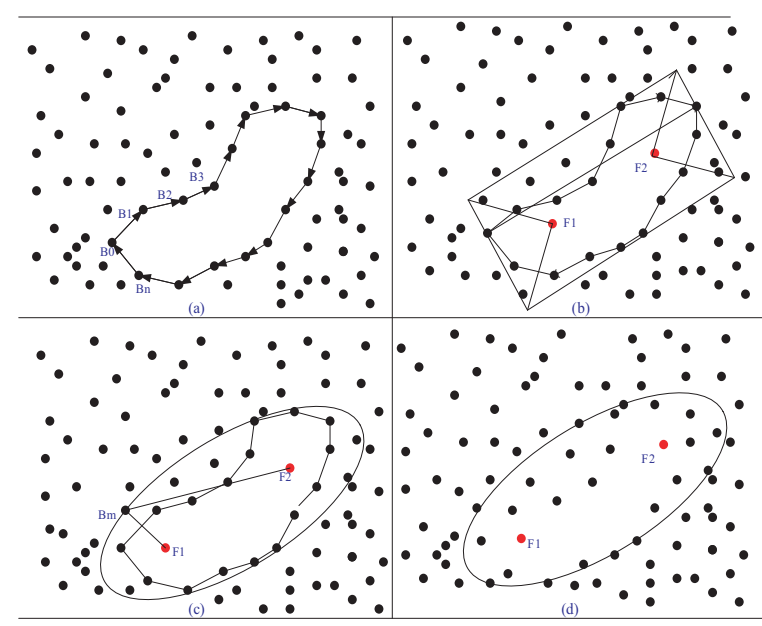

Fig. 3. The process of a hole being reshaped with an ellipse

distributed, that is, a place that has ordinary nodes will have super nodes.

\section{B. Protocol Design}

We assume that the source node knows the location of the destination node via some location service, such as home region-based location service and multiple home regions based location service $[16,17]$, and then can compute the logical location information by using such location information. Here, when routing, we mainly make use of the logical location information in the $\mathrm{CH}$. The grid center can be considered as the logical location of a $\mathrm{CH}$. The change of the logical location of a $\mathrm{CH}$ is less frequent than its geographic location, thus the use of logical location makes the routing path very stable.

The routing protocol defines two types of identifiers: the node identifier (NID) given when the network is initialized, and the grid center identifier (CID) with a pair of numbers denoting the grid code in the $X$ dimension and $Y$ dimension separately.

All nodes know some system parameters: the total number of nodes $N$, network width $W$, network length $L$, the diameter of grid $d$, and the coordinate of the network origin point $\left(x_{0}, y_{0}\right)$. Then the total number of grids is $\frac{W L}{d^{2}}$

Definition 1: Suppose the coordinate of a node is $(X, Y)$, then CID can be labeled by two integers:

$$
\left\lceil\left(X-x_{0}\right) / d\right\rceil,\left\lceil\left(Y-y_{0}\right) / d\right\rceil
$$

(1) Hole-reshaping with ellipse. It is very important to reshape a hole with an ellipse, because it can help us make a decision on which side of the hole to route a packet in order to find a shorter routing path. At present, the literature about reshaping a hole is very little. Here, we give an introduction to the idea from Yu et al [18].

As shown in Fig. 3, all nodes are CHs (cluster members are not shown for clarity). Some adjacent empty virtual grids form a hole. In Fig. 3(a), we assume $B_{0}$ firstly detects that it lies on the boundary of the hole, and then it initiates a HBD packet 
with its ID and forwards the packet to the boundary node $B_{1}$ by the right hand rule [19]. Node $B_{1}$ inserts its location information into the received HBD packet and forwards it to node $B_{2}$ also by the right hand rule. This process repeats until the HBD packet has traveled around the hole and eventually has been received by the initiator node $B_{0}$. $B_{0}$ gets the location information of all boundary nodes of the hole from the received HBD. Then $B_{0}$ selects two nodes $B_{p}$ and $B_{q}$ from $\left\{B_{0}, B_{1}, \ldots, B_{m}\right\}$ so that the distance between $B_{p}$ and $B_{q}$ is the longest distance among distances between any two nodes in $\left\{B_{0}, B_{1}, \ldots, B_{m}\right\}$. Then on each side of $B_{p} B_{q}$, a node with vertical distance from itself to $B_{p} B_{q}$ is longer than other hole boundary nodes on the corresponding side of $B_{p} B_{q}$ e.g., $B_{j}$ and $B_{k}$ are selected by node $B_{0}$, then through $B_{p}, B_{j}, B_{q}$ and $B_{k}, B_{0}$ can obtain a rectangle, and the bisector of the four right angles of the rectangle intersect at point $F_{1}$ and $F_{2}$ with coordinates $\left(x_{1}, y_{1}\right)$ and $\left(x_{2}, y_{2}\right)$ as shown in Fig. 3(b). Then $B_{0}$ selects $B_{m}$ from $\left\{B_{0}, B_{1}, \ldots, B_{m}\right\}$ so that the total distance of $B m F_{1}$ and $B_{m} F_{2}$ is bigger than that of the other nodes in $\left\{B_{0}, B_{1}, \ldots, B_{m}\right\}$ as shown in Fig. 3(c). Based on $\left|F_{1} F_{2}\right|=2 c$ and $\left|F_{1} B_{m}\right|+\left|F_{2} B_{m}\right|=2 a$, we can get an ellipse and reshape the hole with this ellipse as shown in Fig. $3(d)$.

(2) Broadcasting the hole information. When we reshape a hole with an ellipse, we set the time-to-live (TTL) of a broadcast packet with a system parameter $h$. The packet also contains the focus coordinates of the ellipse, the center coordinates of the ellipse, the long axis, and the short axis. The packet will be forwarded to all $\mathrm{CHs}$ in the hole boundary, and then those $\mathrm{CHs}$ broadcast it to other $\mathrm{CHs}$ in their $h$-hop range. Here, the system parameter $h$ denotes the recovery strength of the system, which is the hop count between the farthest $\mathrm{CH}$ and the current $\mathrm{CH}$ with which the information is forwarded. The proper value of $h$ can be determined by the size and the shape of holes.

(3) Hole-bypassing strategy. In this paper, we propose the HRR routing protocol, which mainly solves the following problems: the location error problem due to node mobility, the local optimum problem due to holes, and the triangular routing problem and blind detouring problem in existing hole-avoiding routing protocols. To solve the location error problem, each $\mathrm{CH}$ maintains a routing table, which contains information about all its cluster members and its neighbor $\mathrm{CHs}$. It can strengthen the redundancy about the location error information. To deal with the local optimum problem and the triangular routing problem, we propose a method of holebypassing that pre-collects the information of the hole to avoid the hole in advance. We solve the blind detouring problem by reshaping a hole with an ellipse and making a decision on which side to route packets in order to make a shorter routing path.

The hole-bypassing strategy is described as follows: When a data packet reaches $M$ which contains the hole information, $M$ will judge whether the distance to $D$ is in the direction of the hole or not. If it is, $M$ separately makes two pairs of tangents of the ellipse regarding $M$ and $D$, compares their lengths, and

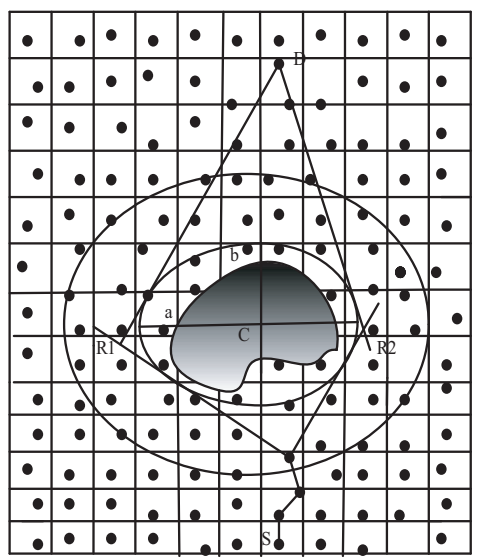

Fig. 4. The determination of the local optimum problem and the direction of the hole

then selects the pair with the shortest path length to forward the data packet by greedy algorithm. Otherwise, $M$ will select a node whose distance to $D$ is shorter than from $M$ and all other neighbor nodes, and then forward the data packet to the node by greedy routing.

In Fig. 4, we use an example to explain the basic idea of the proposed HRR protocol in detail. The inner ellipse denotes a shape with the hole reshaped, and the outer ellipse is the range of the shadow of the hole for 2 hops. We assume $M$ is in the range of the shadow of the hole and its coordinates are $\left(x_{m}, y_{m}\right) . D$ is located at $\left(x_{d}, y_{d}\right)$. There is a hole in the direction from $S$ to $D$, and the hole reshaped is the ellipse as follows:

$$
\frac{\left(x-x_{1}\right)^{2}}{a^{2}}+\frac{\left(y-y_{1}\right)^{2}}{b^{2}}=1
$$

Where $C\left(x_{1}, y_{1}\right)$ is the center coordinates of the ellipse, $a$ is the long axis, and $b$ is the short axis.

The line between $M$ and $D$ is described as follows:

$$
y=\frac{\left(y_{m}-y_{d}\right)}{\left(x_{m}-x_{d}\right)} x-\frac{\left(y_{m}-y_{d}\right)}{\left(x_{m}-x_{d}\right)} x_{m}+y_{m}
$$

Here, the source node $S$ firstly forwards the data packet by greedy algorithm. When the data packet reaches $M$, which is in the range of the shadow of the hole, $M$ decides whether the line from itself to the destination will have two intersection points with the ellipse or not. If there are not two intersection points, it concludes that there is no hole in the direction from $M$ to $D$, and $M$ may forward the data packet to the next hop node by greedy algorithm. Otherwise, $M$ separately makes two pairs of tangents of the ellipse regarding $M$ and $D$. That is $\left(M R_{1}, R_{1} D\right)$ and $\left(M R_{2}, R_{2} D\right)$. Then, we select the pair with the short path length. Here we select the pair $\left(M R_{2}, R_{2} D\right)$. When $M$ forwards the packet, it will send the data packet to $R_{2}$ by greedy algorithm. Finally $R_{2}$ sends the packet to $D$.

In addition, we need to consider two special cases as follows:

(1) The source node $S$ is in the range of the ellipse as shown in Fig. 5 (Left). When $S$ finds itself in the range of the 

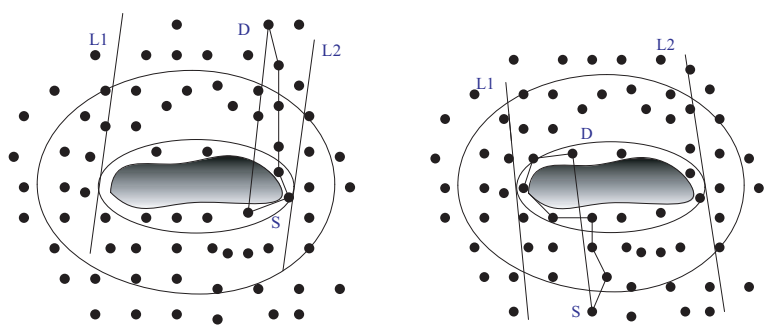

Fig. 5. Two special cases in HRR. Left: $S$ in the range of the ellipse, Right: $D$ in the range of the ellipse

ellipse, it separately makes two tangents of the ellipse, namely, $L_{1}$ and $L_{2}$, which are parallel to $S D$. Then $S$ finds that the line between $S D$ and $L_{1}$ is shorter than the line between $S D$ and $L_{2}$. So, when $S$ forwards the data packet, it will select the shorter path along the right side of $S$, which can be similar to the perimeter routing in GPSR. When the data packet again reaches a node which is in the range of the shadow of the hole, it will forward the data packet by HRR until the data packet gets to the destination node.

(2) The destination node $D$ is in the range of the ellipse as shown in Fig. 5 (Right). First, the data packet is forwarded by HRR. When a node in the range of the shadow of the hole that receives the data packet and finds the destination node is also in the range of the shadow of the hole, it will use the same idea in case (1), and decide along which side of the hole to forward the packet according to the overall hole information.

The HRR protocol is described as follows:

Step 1: When the source node $S$ needs to send data to destination node $D, S$ first gets $D$ 's geographic location information by location service and then calculates its logical location by using Formula (1) according to its geographic location information, that is, it is in the grid.

Step 2: If $S$ is a cluster member, it sends the data to its $\mathrm{CH}$, which will determine whether it is in the shadow of the hole as well as whether there is a local optimum problem between $S$ and $D$. If so, it will avoid the hole by using GPSR protocol and then turn to Step 3; otherwise, it turns to Step 3 directly.

Step3: Source $\mathrm{CH}$ and intermediate $\mathrm{CHs}$ forward the data by greedy routing until the data reaches a $\mathrm{CH}$ that contains the hole information. At this point, the node uses the holebypassing strategy until it reaches the $\mathrm{CH}$ where there is no local optimum problem and goes on the greedy forwarding.

Step 4: Repeat Step 3 until the data arrives at the destination $\mathrm{CH}$.

Step 5: The destination $\mathrm{CH}$ sends the data to node $D$ by its routing table.

We can see that the HRR protocol solves the local optimum problem as well as the triangle routing problem and the blind hole-bypassing problem that exist in GPSR and HSR protocols. In addition, the node that gets the overall information of the hole decides which side of the hole is better to forward the packet according to the overall hole information, and the location of the destination node. HSR only gets the local
Table I

NETWORK SIMULATION PARAMETERS

\begin{tabular}{|l|l|l|l|}
\hline Grid area & Grid number & Maximum speed & Pause time \\
\hline $1,800 \mathrm{~m} \times 1,800 \mathrm{~m}$ & 81 & $10 \mathrm{~m} / \mathrm{s}$ & $5 \mathrm{~s}$ \\
$6,000 \mathrm{~m} \times 6,000 \mathrm{~m}$ & 900 & $10 \mathrm{~m} / \mathrm{s}$ & $5 \mathrm{~s}$ \\
\hline
\end{tabular}

information of the hole so that there will be a certain degree of blindness when it chooses the next hop. As a result, it is very easy to see that our proposed hole-bypassing routing protocol is better than HSR. Below, we will compare HRR, GPSR, and HSR protocols by simulations.

\section{Performance eValuation}

In this section, we evaluate the performance of the proposed protocol by simulations. First we describe our performance metrics and simulation settings. Then we evaluate the system performance by comparing the proposed protocol with GPSR and HSR protocols.

\section{A. Performance Metrics and Simulation Settings}

Nowadays, the perimeter routing strategy in GPSR [20] and HSR are state-of-the-art recovery strategies based on geographic location information. We will compare the proposed protocol (HRR) with GPSR and HSR by simulations and illustrate that HRR is better in terms of routing path length, routing delay, and network scalability.

We assume that the short wireless transmission radius $r$ is $280 \mathrm{~m}$, the side length of each grid is $200 \mathrm{~m}$, and the long wireless transmission radius $R$ is $560 \mathrm{~m}$, which can guarantee that the $\mathrm{CH}$ in the center of a grid can communicate with all its 8 adjacent $\mathrm{CHs}$. The mobile nodes follow the random waypoint model in the simulations. In this paper, simulations are conducted in two sizes of network scales, and the parameter settings are shown in Table 1 . We also use the average path length and the average routing delay as performance evaluation metrics. Average path length refers to the average total hops for all routing paths between pairs of source and destination nodes. Average routing delay refers to the average delay of all routing paths which are successfully built.

\section{B. Simulation Results}

Fig. 6 (Left) shows that the average path length changes over the number of adjacent empty grids in the $1,800 \mathrm{~m} \times 1,800 \mathrm{~m}$ network. Fig. 6 (Right) shows that the average path length changes over the number of adjacent empty grids in the $6,000 \mathrm{~m} \times 6,000 \mathrm{~m}$ network. From Fig. 6, we can see that almost all the nodes take greedy algorithm to forward data packets to the destination node when there is no hole or the hole is relatively small in the three routing protocols, and thus their average path lengths are relatively close to each other. However, when the hole becomes larger, they will meet the hole with increasing probability, and the respective routing protocols' feature will be manifested. GPSR forwards data packets from the hole boundary when it meets the hole. Therefore, GPSR takes the longest path. HSR avoids the hole in advance, but it is aware of only the local information about 

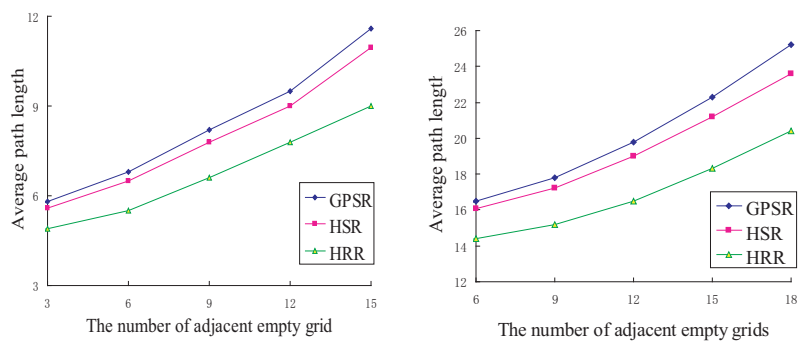

Fig. 6. The average path length. Left: $1,800 \times 1,800$ network area. Right: $6,000 \times 6,000$ network area
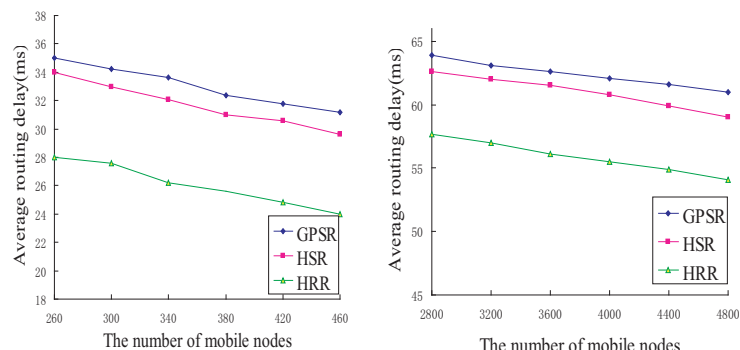

The number of mobile nodes

Fig. 7. The average routing delay. Left: $1,800 \mathrm{~m} \times 1,800 \mathrm{~m}$ area with 6 adjacent empty grids. Right: $6,000 \mathrm{~m} \times 6,000 \mathrm{~m}$ area with 12 adjacent empty grids

the hole, and HRR knows all the information about the hole, and thus can decide which side of the hole will be best when the data packet is forwarded. Therefore, its average path length is shorter than GPSR and HSR. With the increase in the number of holes, HRR will generate a shorter path length than that of GPSR and HSR. Therefore, in a network of the same size, HRR is better than GPSR and HSR.

Figures 7 separately describes the average routing delay changes over two different network scales with 6 adjacent empty grids and 12 adjacent empty grids, respectively. We can see that the average routing delay in HRR protocol is shorter than that in HSR and GPSR. With the increase of each grid node density, there will be more $\mathrm{CHs}$ in each grid and larger range options in the next $\mathrm{CHs}$, and thus the average routing delay will be smaller.

Simulation results show that our proposed routing protocol can effectively solve the local optimum problem, the triangular routing problem, and the blind detouring problem. At the same time, we may see that the proposed protocol also runs well in large-scale mobile networks, such as $6,000 \mathrm{~m} \times 6,000 \mathrm{~m}$ network area.

\section{CONCLUSIONS}

We proposed a novel hole-bypassing model and a logicallocation-based hole-avoiding routing protocol based on the model. It effectively solves the local optimum problem, the triangular routing problem, and the blind detouring problem in large-scale MANETs. Simulation studies show that the proposed protocol outperforms the GPSR and HSR routing protocols due to shorter routing paths and thus a smaller routing delay, which can scale well in large-scale networks. In fact, there are still some problems to be further investigated for our future work, such as choosing the important system parameter $h$, which denotes the recovery strength of the system.

\section{REFERENCES}

[1] C. E. Perkins, E. M. Royer, "Ad Hoc On-Demand Distance Vector Routing," Proc. of the 2nd IEEE Workshop on Mobile Computing Systems and Applications, New Orleans, LA, pp. 90-100, February 1999.

2] D. B. Johnson, D. A. Maltz, "Dynamic Source Routing in Ad Hoc Wireless Networks," Mobile Computing, Vol. 353, Kluwer Academic Publishers, pp. 153-181, 1996.

[3] S. Basagni, I. Chlamtac, V. R. Syrotiuk, and B. A. Woodward, "A Distance Routing Effect Algorithm for Mobility (DREAM)," Proc. of the 4th Annual ACM/IEEE International Conference on Mobile Computing and Networking (MobiCom 1998), pp. 76-84, October 1998.

[4] Y.-B. Ko and N. H. Vaidya, "Location-Aided Routing (LAR) in Mobile Ad hoc Networks," ACM Wireless Networks, 6(4): 307-321, July 2000.

[5] N. Ahmed, S. Kanhere, and S. Jha, "The Holes Problem in Wireless Sensor Networks: A Survey," ACM SIGMOBILE Mobile Computing and Communication Review, 9(2): 4-18, April 2005.

[6] C. Chang, K. Shih, S. Lee, and S. Chang, "RGP: Active Route Guiding Protocol for Wireless Sensor Networks with Obstacles," Proc. of the 3rd IEEE International Conference on Mobile Ad Hoc and Sensor Systems (MASS 2006), pp. 367-376, October 2006.

[7] K. Liu, N. Abu-Ghazaleh, and K. Kang, "Location Verification and Trust Management for Resilient Geographic Routing," Journal of Parallel and Distributed Computing, 67(2): 215-228, February 2007.

[8] X. Wu, G. Chen, and S. Das, "On the Energy Hole Problem of Nonuniform Node Distribution in Wireless Sensor Networks," Proc. of the 3rd IEEE International Conference on Mobile Ad Hoc and Sensor Systems (MASS 2006), pp. 180-187, October 2006.

[9] B. Karp and H.T. Kung, "GPSR: Greedy Perimeter Stateless Routing for Wireless Networks," Proc. of the 6th Annual ACM/IEEE International Conference on Mobile Computing and Networking (MobiCom 2000), pp. 243-254, August 2000.

[10] G. Wang, L. Zhang, and J. Cao, "Hole-Shadowing Routing in LargeScale MANETs," International Journal of Sensor Networks (Inderscience Publishers), 4(4), pp. 220-229, 2008.

[11] C. Liu and J. Wu, "Destination-Region-Based Local Minimum Aware Geometric Routing," Proc. of the 4th IEEE International Conference on Mobile Ad Hoc and Sensor Systems (MASS 2006), pp 1-9, October 2007.

[12] Z. Jiang, J. Ma, W. Lou, and J. Wu, "An Information Model for Geographic Greedy Forwarding in Wireless Ad-Hoc Sensor Networks," Proc. of the 27th IEEE Conference on Computer Communications (INFOCOM 2008), pp. 825-833, April 2008.

[13] K. Gabriel and R. Sokal, "A New Statistical Approach to Geographic Variation Analysis," Systematic Zoology, 18(3): 259-278, September 1969.

[14] T. Godfried and T. Toussain, "The Relative Neighborhood Graph of a Finite Planar Set," Pattern Recognition, 12(4): 261-268, 1980.

[15] J. Na and C. Kim, "GLR: A Novel Geographic Routing Scheme for Large Wireless Ad-Hoc Networks," Computer Networks (Elsevier), 50(17): 3225-3522, December 2006.

[16] M. Fayed and H. T. Mouftah, "Characterizing the Impact of Routing Holes on Geographic Routing," Proc. of the 2005 IEEE International Conference on Systems Communications, pp. 401-406, August 2005.

[17] Q. Fang, J. Gao, and L.J. Guibas, "Locating and Bypassing Routing Holes in Sensor Networks," Proc. of the 23th IEEE Conference on Computer Communications (INFOCOM 2004), pp.2458-2468, March 2004.

[18] F. Yu, E. Lee, Y. Choi, S. Park, D. Lee, Y. Tian, and S. Kim, "A Modeling for Hole Problem in Wireless Sensor Networks," Proc. of the 2007 International Conference on Wireless Communications and Mobile Computing (IWCMC 2007), Honolulu, Hawaii, USA, pp.12-16, August 2007.

[19] J. A. Bondy and U. S. R. Murty, "Graph Theory with Applications," Elsevier North-Holland, 1976.

[20] M. Mauve, J. Widmer, and H. Hartenstein, "A Survey on Position-Based Routing in Mobile Ad Hoc Networks," IEEE Network Magazine, 15(6): 30-39, Nov./Dec. 2001. 